\title{
Síndrome da íris flácida como complicação do uso do bloqueador alfadrenérgico para tratamento de hiperplasia prostática benigna
}

Floppy iris syndrome as a complication of the use of an alpha-adrenergic blocker for the treatment of benign prostatic hyperplasia

Síndrome del iris flácido como complicación del uso de un bloqueador alfa-adrenérgico para el tratamiento de la hiperplasia prostática benigna

Izabely Lima Assunção ORCID: https://orcid.org/0000-0001-8262-9192

Universidade CEUMA, Brasil

E-mail: izabelyyyyy@gmail.com

Ana Karoline de Almeida Mendes ORCID: https://orcid.org/0000-0003-2654-7859 Universidade CEUMA, Brasil

E-mail: anakarolinemendesal@hotmail.com

Gustavo Soares Gomes Barros Fonseca ORCID: https://orcid.org/0000-0002-7287-1409 Universidade CEUMA, Brasil E-mail: gustavosgbf@gmail.com

Lara Vasconcelos Normando ORCID: https://orcid.org/0000-0002-5649-9416 Universidade CEUMA, Brasil E-mail: laranormandoo@gmail.com Felipe de Queiroz Leite ORCID: https://orcid.org/0000-0002-7422-4483 Universidade CEUMA, Brasil

E-mail: felipeleite.adv@hotmail.com

Lise Feitosa Novais Miranda ORCID: https://orcid.org/0000-0001-7804-2444 Universidade CEUMA, Brasil E-mail: lise_med@outlook.com Pryscilla Oliveira Melo ORCID: https://orcid.org/0000-0002-6215-5644 Universidade CEUMA, Brasil

E-mail: pryscillameloo@ hotmail.com Nikole Sousa Dias

ORCID: https://orcid.org/0000-0003-1818-8788 Universidade CEUMA, Brasil

E-mail: sousanikole@gmail.com

Valéria Marques da Silva ORCID: https://orcid.org/0000-0003-3719-7786 Universidade CEUMA, Brasil

E-mail: valeriamarques_med@hotmail.com

Rebeca Xavier Fontes Cunha ORCID: https://orcid.org/0000-0001-9131-2859 Universidade CEUMA, Brasil

E-mail:rebecaxavier@gmail.com

Giovanna de Paiva Adler

ORCID: https://orcid.org/0000-0002-0688-763X Universidade CEUMA, Brasil

E-mail: Giadler2003@gmail.com Raissa de Sousa Nunes ORCID: https://orcid.org/0000-0002-7022-9674 Universidade CEUMA, Brasil E-mail:raisssa@gmail.com

João Victor Carvalho da Paz ORCID: https://orcid.org/0000-0002-2896-1079 Universidade CEUMA, Brasil

E-mail:joaovictorpaz@gmail.com 
Ana Letícia Lopes Abreu Silva

ORCID: https://orcid.org/0000-0003-3210-5424 Universidade CEUMA, Brasil

E-mail:analeticialopes@gmail.com

Marcos Vijano da Silva Souza

ORCID: https://orcid.org/0000-0003-1156-3258 Universidade CEUMA, Brasil

E-mail: marcosvijanoo@ hotmail.com

Arthur Brício Silva Costa

ORCID: https://orcid.org/0000-0003-3775-0262 Universidade CEUMA, Brasil

E-mail: arturbricioo@gmail.com

David Brusaca Abreu

ORCID: https://orcid.org/0000-0001-8296-4492 Universidade CEUMA, Brasil E-mail: david.bba@gmail.com

Sarah Barreto Ramos Pereira ORCID: https://orcid.org/0000-0002-1323-3298 Universidade CEUMA, Brasil

E-mail:sarah@gmail.com

Fernanda Nogueira Barreto

ORCID: https://orcid.org/0000-0001-5836-8105 Universidade CEUMA, Brasil

E-mail: nandanbarreto@gmail.com

Claudia Nery do Nascimento Coelho

ORCID: https://orcid.org/0000-0002-7739-1885 Universidade CEUMA, Brasil

E-mail: claudia.nc@hotmail.com

Chelsea Magalhães Tavares da Silva

ORCID: https://orcid.org/0000-0003-1018-1717 Universidade CEUMA, Brasil

E-mail: magalhaes.chelsea@gmail.com

Wellington Ubaldino de Freitas Júnior

ORCID: https://orcid.org/0000-0002-3513-539X Universidade CEUMA, Brasil

E-mail: wjuninhoo_@outlook.com

\title{
Resumo
}

A hiperplasia prostática benigna (HBP) é uma alteração urinária associada a sintomas no trato urinário inferior, os quais podem ser urgência miccional, polaciúria, esforço miccional e tenesmo vesical. Os alfabetabloqueadores, utilizados no tratamento medicamentoso em alguns casos de HBP, podem causar uma inibição da musculatura responsável pela dilatação da íris, ocorrendo o desenvolvimento da síndrome da Síndrome da Íris Intraoperatória (SIFI). Dessa maneira, o objetivo da seguinte revisão foi relacionar os antagonistas de receptores alfa 1 adrenérgicos no tratamento da HBP com desenvolvendo da SIFI. Trata-se de uma revisão bibliográfica sistemática que utilizou as plataformas PubMed, sciELO e Google Scholar, como base de dados para pesquisa de artigos científico, com recorte temporal entre 2016 e 2021, na língua inglesa. De acordo com o mecanismo de busca, foram encontrados 10 resultados, sendo 10 artigos na base de dados PubMed e Google Scholar e 0 na base SciELO. Constatou-se, portanto, de acordo com as literaturas analisadas a relação entre uso de antagonista do receptor alfa-1 adrenérgico, em especial a tansulosina, para tratamento da HBP e o desenvolvimento de SIFI.

Palavras-chave: Hiperplasia prostática benigna; Síndrome da íris flácida intraoperatória; Antagonistas de receptores alfa 1 adrenérgicos.

\begin{abstract}
Benign prostatic hyperplasia $(\mathrm{BPH})$ is a urinary alteration associated with symptoms in the lower urinary tract, which can be voiding urgency, pollakiuria, voiding effort and bladder tenesmus. Meta-blockers, used in drug treatment in some cases of BPH, can inhibit the muscles responsible for iris dilation, leading to the development of alphab intraoperative iris syndrome (SIFI). Thus, the aim of the following review was to relate alpha 1 adrenergic receptor antagonists in the treatment of BPH with the development of SIFI. This is a systematic literature review that uses the platforms PubMed, sciELO and Google Scholar as a database for researching scientific articles, with a time frame between 2016 and 2021 , in English. According to the search engine, 10 results were found, 10 articles in the PubMed and Google Scholar databases and 0 in the SciELO database. Therefore, according to the literature analyzed, the relationship between the use of alpha-1 adrenergic receptor antagonists, especially tamsulosin, for the treatment of BPH and the development of SIFI was found.
\end{abstract}

Keywords: Benign prostatic hyperplasia; Intraoperative flaccid iris syndrome; Alpha 1 adrenergic receptor antagonists. 


\section{Resumen}

La hiperplasia prostática benigna (HBP) es una alteración urinaria asociada a síntomas en el tracto urinario inferior, que pueden ser urgencia miccional, polaquiuria, esfuerzo miccional y tenesmo vesical. Los metabloqueantes, utilizados en el tratamiento farmacológico en algunos casos de HPB, pueden inhibir los músculos responsables de la dilatación del iris, lo que conduce al desarrollo del síndrome de iris intraoperatorio alfab (SIFI). Por lo tanto, el objetivo de la siguiente revisión fue relacionar los antagonistas del receptor adrenérgico alfa 1 en el tratamiento de la BPH con el desarrollo de SIFI. Se trata de una revisión sistemática de la literatura que utiliza las plataformas PubMed, sciELO y Google Scholar como base de datos para la investigación de artículos científicos, con un marco temporal entre 2016 y 2021, en inglés. Según el buscador, se encontraron 10 resultados, 10 artículos en las bases de datos PubMed y Google Scholar y 0 en la base de datos SciELO. Por tanto, según la literatura analizada, se encontró la relación entre el uso de antagonistas de los receptores alfa-1 adrenérgicos, especialmente tamsulosina, para el tratamiento de la HBP y el desarrollo de SIFI.

Palabras clave: Benign prostatic hyperplasia; Intraoperative flaccid iris syndrome; Alpha 1 adrenergic receptor antagonists.

\section{Introdução}

A hiperplasia benigna da próstata (HBP) é uma alteração urinária que acomete homens geralmente a partir dos 40 anos, atingindo metade da população masculina na quinta década de vida, sendo associada a sintomas no trato urinário inferior (Arruda \& Arruda, 2010). Essa condição ao acometer o ato de urinar, coloca em risco a qualidade de vida desses pacientes, interferindo nas atividades diárias e no padrão de sono, podendo levar a retenção urinária, hidronefrose e insuficiência renal (Averbeck et al., 2010).

O quadro clínico do paciente que apresenta HPB é variável, de forma que o indivíduo pode apresentar sintomas intermitentes ou progressivos (Dib, 2003). Os sintomas são divididos em três grupos: sintomas de armazenamento (urgência miccional; polaciúria; nictúria), sintomas de esvaziamento (esforço miccional, sensação de esvaziamento incompleto) e queixas pós-miccionais (tenesmo vesical e gotejamento pós-miccional (Rizzi \& Rigo, 2021).

O diagnóstico clínico é baseado na presença de sintomas no trato urinário inferior e exame digital de próstata. No que tange ao tratamento, as principais opções de manejo são: expectante (conversador) e medicamentoso (Lopes \& Tavares, 2005). No tratamento conservador algumas medidas comportamentais ajudam a reduzir os sintomas, como diminuir ingesta de líquidos, exercícios para treinamento vesical, redução do uso de álcool, café e cigarro. Já sobre o tratamento medicamentoso, o uso de fármacos da classe alfa bloqueadores causam benefício imediatos dos sintomas (Batlouni \& Albuquerque, 2000).

Para pacientes com sintomas obstrutivos leves a moderados, os bloqueadores alfas adrenérgicos (terazosina, doxasosina, tansulosina, alfuzosina) podem melhorar a micção, de forma que relaxam os músculos da próstata e da saída da bexiga, melhorando o fluxo da urina (Oliveira, Rodrigues, \& Paula, 2007).

As reações adversas dos betabloqueadores são efeitos colaterais descritos como fadiga, depressão, capacidade de exercício diminuída, disfunção sexual e crises de asma (Bortolotto \& Consolim-Colombo, 2009). Os betabloqueadores, também, têm sido relacionados a efeitos metabólicos indesejáveis que podem influenciar a evolução do paciente para um quadro de hipertensão arterial, além de diabetes. No caso de pacientes com hiperplasia prostática benigna um dos efeitos colaterais pode ser o desenvolvimento Síndrome da Íris Intraoperatória (SIFI) (Robertson \& Biaggioni, 2017).

Os alfabloqueadores podem ocasionar a inibição da musculatura responsável pela dilatação da íris ocorrendo assim o desenvolvimento da síndrome, a qual afeta com mais frequência homens obesos, caracterizada por pálpebras superiores flácidas associadas a conjuntivite papilar crônica (Averbeck et al., 2010; Plavnik, 2002).

Dessa forma, o objetivo da seguinte revisão bibliográfica foi relacionar os antagonistas de receptores alfa 1 adrenérgico no tratamento da hiperplasia benigna prostática com desenvolvimento da SIFI. 


\section{Metodologia}

Trata-se de uma revisão bibliográfica sistemática, do tipo quantitativa, que utilizou as plataformas PubMed, Scientific Eletronic Library On-line (SciELO) e Google Scholar como base de dados para pesquisa dos artigos científicos. Foram utilizadas literaturas publicadas com recorte temporal de 2016 a 2021, na língua inglesa, que abordavam a relação dos antagonistas do receptor alfa-1 adrenérgico para o tratamento de hiperplasia benigna prostática com o desenvolvimento da Síndrome da Íris Flácida Intraoperatória ("Floppy Iris Syndrome").

Os descritores utilizados seguiram a descrição dos termos DeCs (Descritores em Saúde) e Medical Subject Headings (MeSH) no idioma inglês, como mostra a Tabela 1.

Quadro 1. Estratégia de busca para o estudo.
$\begin{aligned} & \text { ((("Alpha blocker"[All Fields] OR "alpha antagonist"[All Fields]) AND "floppy iris"[All Fields]) OR "floppy } \\ & \text { iris syndrome"[All Fields]) AND ("benign prostatic hyperplasia"[All Fields]) }\end{aligned}$

Fonte: Autores.

Nesta revisão, os critérios de exclusão utilizados foram: Livros, documentos de projetos de dissertação, resumos em eventos, editoriais, revisões de literatura, relatos de caso isolados, artigos que não cumpriam os critérios de inclusão e artigos duplicados.

\section{Resultados e Discussão}

A escolha dos artigos a serem utilizados nesta revisão foi realizada por meio da leitura do título, resumo e, por fim, da leitura do artigo na íntegra, sendo realizada uma análise criteriosa dos artigos fundamentados nos critérios de inclusão e exclusão supracitados.

De acordo com o mecanismo de busca, foram encontrados 10 resultados, sendo 10 artigos na base de dados PubMed e Google Scholar e 0 na base SciELO (Quadro 1).

Dos 10 resultados obtidos, 6 foram excluídos com base nos critérios de exclusão para a pesquisa e, além disso, não abordavam de forma objetiva sobre o tema, sendo apenas 4 artigos utilizados na confecção do estudo. Todos os artigos escolhidos serão expostos no Quadro 1, seguindo a ordem de ano de sua publicação. 
Quadro 2 - Artigos selecionados nas bases de dados PubMed e Google Scholar.

\begin{tabular}{|c|c|c|}
\hline AUTOR/ANO & TÍTULO & OBSERVAÇÕES \\
\hline (Lunacek et al., 2018) & $\begin{array}{l}\text { Ten years of intraoperative } \\
\text { floppy iris syndrome in the era } \\
\text { of } \alpha \text {-blockers }\end{array}$ & $\begin{array}{l}\text { O tratamento sistemático com tansulosina é um desafio entre os } \\
\text { oftalmologistas, com risco de complicações. A modificação da dose é } \\
\text { capaz de reduzir a incidência da síndrome da íris flácida intraoperatória. }\end{array}$ \\
\hline $\begin{array}{l}\text { (Christou, } \\
\text { Tsinopoulos, Ziakas, } \\
\text { \& Tzamalis, 2020) }\end{array}$ & $\begin{array}{l}\text { Intraoperative Floppy Iris } \\
\text { Syndrome: Updated } \\
\text { Perspectives }\end{array}$ & $\begin{array}{l}\text { Oftalmologistas e urologistas devem cooperar para educar uns aos outros } \\
\text { sobre os riscos de suas respectivas áreas, visto que é primordial que a } \\
\text { tansulosina seja administrada apenas após a facoemulsificação ou o uso } \\
\text { de um ARA al não seletivo para o tratamento da HPB. Ademais, é } \\
\text { crucial que se tenha conhecimento por parte dos profissionais acerca dos } \\
\text { fatores de risco associados à SIFI. }\end{array}$ \\
\hline $\begin{array}{l}\text { (Yang, Liu, Fan, } \\
\text { Grzybowski, \& } \\
\text { Wang, 2020) }\end{array}$ & $\begin{array}{lcr}\text { A narrative } & \text { review } & \text { of } \\
\text { intraoperative } & \text { floppy } & \text { iris } \\
\text { syndrome: } \text { an update } 2020\end{array}$ & $\begin{array}{l}\text { Os principais fatores de risco associados à SIFI são a idade, sexo, } \\
\text { hipertensão, comprimento axial do olho, inibidores da } 5 \alpha \text {-redutase, outro } \\
\text { antagonista do adrenoceptor } \alpha \text { e neuromoduladores. Ademais, caso a } \\
\text { SIFI ocorra durante a cirurgia de facoemulsificação, complicações como } \\
\text { perda endotelial da córnea, pressão intraocular elevada, perda de vítreo, } \\
\text { trauma da íris, ruptura da cápsula posterior aumentam } \\
\text { significativamente. Faz-se necessário, portanto, avaliar o pré-operatório } \\
\text { de pacientes de alto risco e intervir no intra-operatório para evitar } \\
\text { complicações graves. }\end{array}$ \\
\hline $\begin{array}{c}\text { (Ozcura \& Irgat, } \\
2020)\end{array}$ & $\begin{array}{l}\text { Bilateral Intraoperative Floppy } \\
\text { Iris Syndrome Associated with } \\
\text { Silodosin Intake }\end{array}$ & $\begin{array}{l}\text { Neste relato de caso, um homem de } 63 \text { anos apresentou diminuição da } \\
\text { visão bilateralmente, cujo exame oftálmico revelou catarata nuclear } \\
\text { bilateral. Ele fazia uso da silodosina para tratamento da HPB há um mês. }\end{array}$ \\
\hline
\end{tabular}

Fonte: Autores.

De acordo com a análise bibliográfica, foi possível observar que há uma relação efetiva entre o uso de antagonistas do receptor alfa-1 adrenérgico para o tratamento de hiperplasia benigna prostática e o desenvolvimento da Síndrome da Íris Flácida Intraoperatória (SIFI) (Christou et al., 2020; Lunacek et al., 2018). Essa relação ocorre visto que esses antagonistas atuam inibindo o músculo dilatador da íris, podendo provocar consequências sérias em pacientes que necessitam de cirurgia para correção de catarata posteriormente (Lunacek et al., 2018).

A hiperplasia prostática benigna (HPB) é uma doença que acomete 3 a cada 4 homens acima dos 70 anos, provocando sintomas do trato urinário inferior que afetam a qualidade de vida do paciente. A HPB ocorre porque a próstata tende a aumentar de volume conforme a idade vai aumentando e, por aumentar a tensão sobre o canal da uretra, desencadeia sintomas como fluxo urinário diminuído, gotejamento após urinar, incontinência urinária, nictúria, e até mesmo a sensação de esvaziamento incompleto da bexiga (Lunacek et al., 2018).

Com o objetivo de reduzir a tensão da musculatura da próstata e da uretra, e com isso permitir o fluxo urinário adequado, foram desenvolvidos medicamentos que atuam relaxando a musculatura lisa da próstata e da parede da bexiga, os denominados alfabloqueadores. Dentre essas medicações, podemos citar: a tansulosina, que é alfabloqueador seletivo, e a terozosina, a doxazosina e a alfuzosina, que são não seletivos (Lunacek et al., 2018).

Segundo as literaturas analisadas, os alfabloqueadores, além de agir na musculatura lisa da próstata e da bexiga, também atuam inibindo a musculatura responsável pela dilatação da íris, o que pode resultar a longo prazo em prolapso e constrição da pupila e, em casos de necessidade de correção da catarata, no desenvolvimento da SIFI, que é uma síndrome resultante de uma complicação intraoperatória (Lunacek et al., 2018).

Algumas pesquisas realizadas posteriormente têm demonstrado que o uso de alfabloqueadores não seletivos são preferenciais no tratamento da HPB. Isso porque por ser um inibidor seletivo $\alpha 1-1 \mathrm{~A}$, a tansulosina possui cerca de dez vezes mais afinidade que as outras medicações utilizadas, relatando um efeito mais rápido (Christou et al., 2020).

Um outro ponto a se considerar na relação entre o uso da tansulosina e o desenvolvimento da SIFI é o fato de que essa medicação possui uma meia vida longa de cerca de 10 a 14 dias, além de ficar acumulado em grânulos de pigmento da íris, o que aumenta significativamente as chances de consequências intraoperatórias durante a cirurgia de catarata (Christou et al., 2020; Lunacek et al., 2018). Foi um assunto discutido entre oftalmologistas que a ingestão dessa medicação a longo prazo poderia 
provocar variações anatômicas permanentes, e que mesmo com sua descontinuação não retornariam ao padrão anatômico (Christou et al., 2020). No entanto, há estudos que sugerem que a modificação da dose usual é capaz de reduzir a incidência da SIFI, como demonstrado em pacientes no Japão (González, Muñoz, Lozano, \& Fernández, 2013).

Um novo bloqueador de adrenoceptores alfa 1 mais seletivo e específico para o trato urinário tem sido utilizado como alternativa para a tansulosina, com o intuito de reduzir os riscos à SIFI, no entanto, foi verificado que, apesar de ter menos efeitos colaterais que outros bloqueadores alfa, a silodosina ainda pode estar associada ao seu desenvolvimento, tendo sido relatada raras vezes na literatura (Ozcura \& Irgat, 2020).

Dentre as alterações incluídas na Síndrome da Íris Flácida Intraoperatória, pode-se incluir um estroma flácido da íris que ondula durante a cirurgia, uma miose intraoperatória progressiva, e a tendência do estroma da íris ao prolapso durante a incisão cirúrgica (Lunacek et al., 2018; Yang et al., 2020). Assim, segundo estudos mais recentes, é de fundamental importância que a tansulosina seja administrada apenas após a facoemulsificação ou o uso de um ARA a1 não seletivo para o tratamento da HPB (Christou et al., 2020).

Nessa perspectiva, percebe-se a necessidade da abordagem multiprofissional no tratamento de algumas doenças, visto que o uso de determinadas medicações pode afetar diferentes receptores por todo o corpo, e provocar consequências sérias na vida do paciente. Neste caso, o ideal é que antes de o paciente iniciar o tratamento da HPB com alfabloqueadores, o urologista ou clínico geral faça um encaminhamento ao oftalmologista para realizar uma avaliação ocular pré-tratamento, buscando analisar a melhor terapia para o paciente sem prejudica-lo futuramente, ainda mais considerando-se que a catarata é uma doença bastante prevalente em idosos (Christou et al., 2020; Lunacek et al., 2018).

Segundo a Organização Mundial da Saúde (OMS), até 30\% dos idosos sofre de catarata, e uma parcela significativa desses pacientes é submetida a tratamento cirúrgico para correção todos os anos (Lunacek et al., 2018). Sendo assim, o uso de antagonistas do receptor alfa-1 adrenérgico deve ser bem avaliado quantos aos seus possíveis riscos no paciente antes de ser indicado.

Dentre os principais fatores de risco associados à SIFI estão incluídos: a idade, sexo, hipertensão, comprimento axial do olho, uso de inibidores da $5 \alpha$-redutase, outro antagonista do adrenoceptor $\alpha$ e neuromoduladores. Dessa forma, avaliar o préoperatório dos pacientes de forma cuidadosa é essencial à melhor conduta, visto que caso essa síndrome ocorra durante a cirurgia, algumas complicações, como perda endotelial da córnea, pressão intraocular elevada, perda de vítreo, trauma da íris, e ruptura da cápsula posterior podem se tornar presentes e afetar sua qualidade de vida (Yang et al., 2020).

\section{Conclusão}

De acordo com as literaturas analisadas, tornou-se perceptível a elevada relação entre o uso de antagonistas do receptor alfa-1 adrenérgico, em especial da tansulosina, para o tratamento de hiperplasia benigna prostática e o desenvolvimento da Síndrome da Íris Intraoperatória. Dessa forma, médicos urologistas e clínicos gerais devem permanecer vigilantes sobre os riscos que essas medicações utilizadas no tratamento da HPB podem provocar no paciente, sendo de fundamental importância que atuem em conjunto com oftalmologistas no sentido de indicar avaliação ocular pré-tratamento.

Assim, pode-se perceber que o conhecimento, a antecipação e o reconhecimento dessa síndrome podem levar a menor incidência de complicações cirúrgicas, sendo fundamental a abordagem multiprofissional para melhor conduta.

\section{Referências}

Arruda, P., \& Arruda, J. (2010). A importância do clínico no diagnóstico das doenças da próstata. Proclim, 10, 61-107.

Averbeck, M. A., Blaya, R., Seben, R. R., Lima, N. d., Denardin, D., Fornari, A., \& Rhoden, E. L. (2010). Diagnóstico e tratamento da hiperplasia benigna da próstata. Revista da AMRIGS, 54(4), 471-477. 
Batlouni, M., \& Albuquerque, D. d. (2000). Bloqueadores beta adrenérgicos na insuficiência cardíaca. Arq Bras Cardiol, 75(4), 339-349.

Bortolotto, L. A., \& Consolim-Colombo, F. M. (2009). Betabloqueadores adrenérgicos. Rev Bras Hipertens, 16(4), $215-220$.

Christou, C. D., Tsinopoulos, I., Ziakas, N., \& Tzamalis, A. (2020). Intraoperative Floppy Iris Syndrome: Updated Perspectives. Clinical Ophthalmology (Auckland, NZ), 14, 463.

Dib, P. T. (2003). Resultados do estudo urodinâmico em pacientes diabéticos com sintomas do trato urinário inferior e aumento prostático. Universidade de São Paulo.

González, M.-M. J., Muñoz, N. F., Lozano, E. I., \& Fernández, M. Y. (2013). Intraoperative floppy-iris syndrome. Archivos de la Sociedad Espanola de Oftalmologia, 88(2), 64 .

Lopes, H. V., \& Tavares, W. (2005). Diagnóstico das infecções do trato urinário. Revista da Associação Médica Brasileira, 51(6), 306-308.

Lunacek, A., Al-Ali, B. M., Radmayr, C., Weber, M., Horninger, W., Findl, O., \& Plas, E. (2018). Ten years of intraoperative floppy iris syndrome in the era of $\alpha$-blockers. Central European journal of urology, 71(1), 98.

Oliveira, K. A. C., Rodrigues, A. B. C., \& Paula, A. B. (2007). Técnicas fisioterapêuticas no tratamento e prevenção da incontinência urinária de esforço na mulher. Revista eletrônica F@ pciência, 1(1),31-40.

Ozcura, F., \& Irgat, S. G. (2020). Bilateral intraoperative floppy iris syndrome associated with silodosin intake. The Eurasian journal of medicine, 52(1), 100.

Plavnik, F. L. (2002). Hipertensão arterial induzida por drogas: como detectar e tratar. Rev Bras Hipertens, 9(2), $185-191$.

Rizzi, L. O. C., \& Rigo, R. S. M. (2021). O impacto da hiperatividade detrusora no quadro sintomático do paciente com hiperplasia prostática benigna.

Robertson, D., \& Biaggioni, I. (2017). Fármacos antagonistas de adrenoceptores. Farmacologia Básica e Clínica-13, 152.

Yang, X., Liu, Z., Fan, Z., Grzybowski, A., \& Wang, N. (2020). A narrative review of intraoperative floppy iris syndrome: an update 2020. Annals of Translational Medicine, $8(22)$. 